\title{
Design and Implementation of Online Booking System of University Sports Venues
}

\author{
Can $\mathrm{Li}^{*}$, Junjie Li, Hongxiang Cao and Zhan Meng \\ Management Engineering School, Zhengzhou University, Zhengzhou, China \\ Corresponding Email: 365083792@qq.com
}

\begin{abstract}
To solve the imbalanced use of university sports venues, the online booking system of university sports venues based on the table tennis hall of Zhengzhou University is designed, combining the experience of the existing online booking systems, the main methods and the major ideal of building these systems both at home and aboard. The basic functions of the system are designed according to the general requirement analysis, including user's registration and login, online venue booking, online payment, personal center, message board and database construction. The development of the system used Java programming language, JSP (a Web platform development technology), MySQL database processing technology, JDBC data access model, MyEclipse development platform and Tomcat server. The system has accomplished such functions as online booking, online payment and online message. The tests of the system are run in good conditions. The use of the system has made up for the current luck of sports venues management, solved the problems of the online booking function of university sports venues, improved the efficiency of the venues and meet the needs of efficient use of the venues. The system can be an experience of the management of the other college sports venues.
\end{abstract}

\section{Introduction}

College sports venues are not only an essential part of physical education, but also an important place for fitness activities of the faculties, students as well as nearly residents. At present, sports venues of Zhengzhou University has such problems as places idled, venues are fulled at the high time of movement, which greatly affects the practical efficiency of sports venues resources and increases the management burden to venues. With the rapid development of information technology, college sports venues realizing online booking has become a new trend.

\section{Research status and significance}

\subsection{Research status}

Many venues used the reservation machines to make a booking in Shanghai World Expo in 2010. The State Grid Pavilion and other venues also launched online booking systems [1]. Dai Xiaojing and other scholars designed a sports venue booking system based on .NET [2], which has realized the basic function of venues online booking. They also developed a booking and detection system of sports venues, based on the three-tier architecture theory of the Internet of things(IOT) [3], but it failed to make detailed demands analysis and function design. Wei Honglei's article about the network service management platform system of college sports venues involved in a module of reservation center system [4], which has realized the function of users to booking venues at anytime. Zhang Huai and other scholars also covers a booking module in the design of a stadium management system [5].

Overall, current literature related to this study are less. Moreover, the design schemes of reservation system and realization technology are various. The imbalanced use of college sports venues resources has not been completely solved now. So lots of researches about the online booking system of college sports venues are seriously needed.

\subsection{Research significance}


People can know the usage of every sports venues in Zhengzhou university online, and book any places they need, which can reduce the imbalanced problems that there are no usable places or all the places are being used fully, etc. To a certain extent it can reduce the complicated human affairs, optimize resources management and reduce the imbalanced use of venues.

\section{Requirements analysis and research methods}

\subsection{Requirement analysis}

Many college sports venues are opened for free. But because the usage of venues can't be known good, people often encounter that students of athletics are in the class or there is no place for sports when go to the venues directly. So a online booking system for college sports venues is necessary. The venue booking system should provide humanized interface and complete functions. The basic functions are as follows:

(1) Identity authentication of users: For faculty and students, work number and student Id can respectively be used as an account, which are based on our campus card. Moreover, For the nearly residents, they need register at first, and then input legitimate user name and password. Eventually, after the certification they can booking venues.

(2) Classification of administrator users and ordinary users: Users are divided into administrators users and ordinary users. The administrators have more authorities, such as the management of users, place orders, changing information of places and the bulletin-board.

(3) Real-time information display for venues: Visual graphics based on the actual geographical position of venues need to be built. Users can know the current usage of every place of every venues of our college and can know the situation of the venue booking in the next seven days.

(4) Venue booking and cancel: Users can booking venues in advance in the next week. In order to cater to the teaching time, a day can be divided into fixed period of time for the user to make a order. The user can cancel the order if it hasn't meet the time.

(5) Pay the cost for booking: To standardize the process of the user to make a booking and to reduce the damages due to users go back on their word, payment for booking can be realized by deducting the virtual money. The payment success, the booking success.

(6) Message board: When submit orders, users can leave a message to the administrator if they have more demands.

(7) Personal center module: In this place, users can view their order and balance, and they can modify their personal information as well as password.

(8) Database: It mainly includes the following table: users' information table, venues' information table, booking orders table, opening schedule of venues, etc.

\subsection{Research methods}

(1)Technical route:

The development of system use JSP (Web platform development technology) + JAVA (programming language) + MySQL (database) + JDBC (data access model) [6]+ Javascript (scripting language) as a technical support.

(2)Development tools and environment of the system:

1> Development technology:

Development technology of dynamic webpages: JSP + DIV + CSS ( Javascript, JAVA, JDBC) [7]

2> System development environment:

Operating system: Windows XP Pro3

Software: MyEclipse (tools) [8, 9]

Tomcat6.0(server) [10]

MySQL 5.0 (database)

PhotoshopCS3 (be used to make pictures)

$3>$ System running environment

The server side:

Operating system: Windows 2000 Server

Web server: Tomcat6.0

Web database: MySQL 5.0

Client:

Operating system: Windows 98 / xp and above

The browser version: IE6, IE7 ,IE8 browser, Internet explorer, firefox, Google, Opera, etc.

\section{Design and implementation of the system}

\subsection{Design of the interface}


This design is based on two design concept:

(1) Simple design concept:The purpose of development of this system is to provide convenience to users when they booking venues, and to avoid flashy, so the system' interface is simple but contain complete functions.

(2) Humanized design concept: In the process of design the system, questions are pondered from the perspective of users in order to provide better service for users.

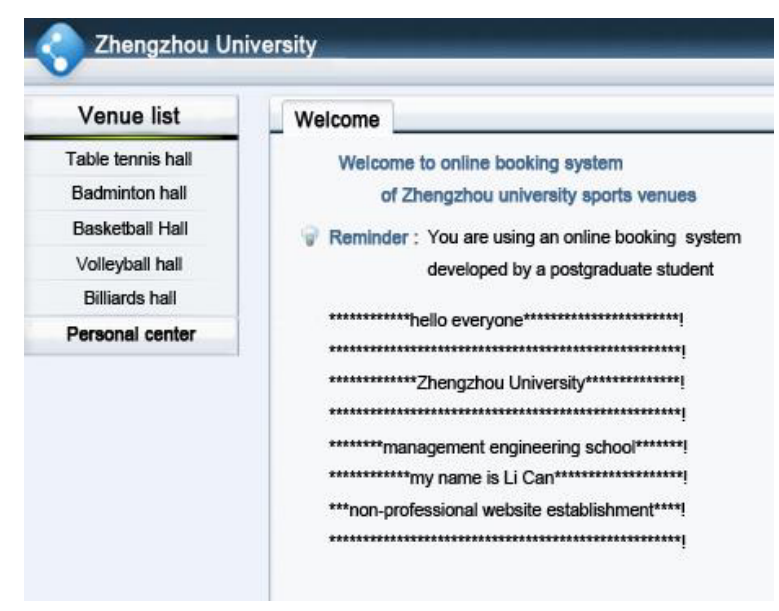

Fig. 1. The welcome screen.

\subsection{Design and implementation of registered and login module}

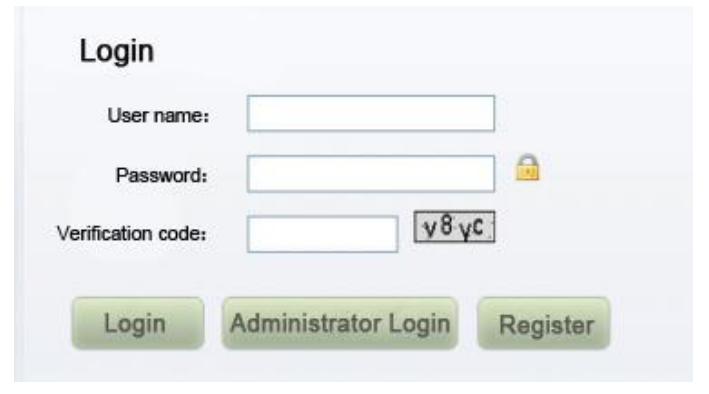

Fig. 2. User login.

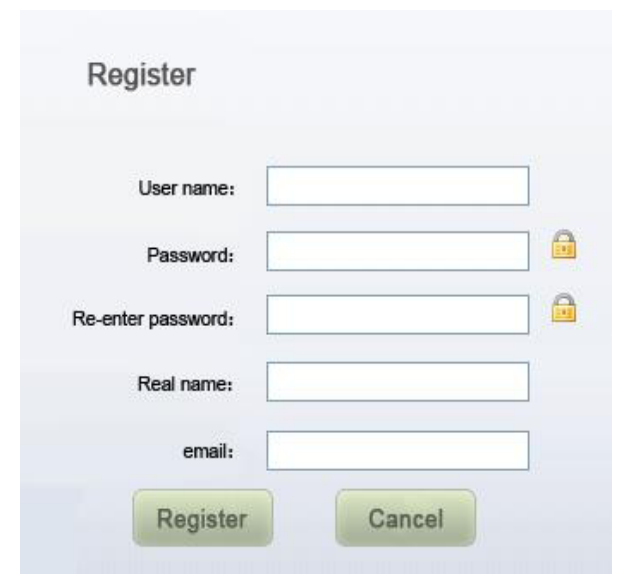

Fig. 3. User registration. 
Users are divided into two categories, they are administrator users and members, Their functions and permissions are as follows:

\begin{tabular}{|c|l|l|}
\hline \multirow{4}{*}{ User } & \multirow{4}{*}{ Members } & Browse the real-time information of the fields \\
\cline { 3 - 3 } classification & & Venue booking and cancel the order \\
\cline { 3 - 3 } and their & & Check their own orders \\
\cline { 3 - 3 } functions & \multirow{4}{*}{$\begin{array}{c}\text { Administrator } \\
\text { users }\end{array}$} & Change their personal information \\
\cline { 3 - 3 } & & Change and delete the board information \\
\cline { 3 - 3 } & & Crowse the real-time information of the fields \\
\hline & & Add or remove and delete all the booking orders \\
\hline
\end{tabular}

Fig. 4. Classification and functions of users.

\subsection{Venue booking function}

Venue booking is the core module of the system and the design of the venue booking process is also an important content of this study. Through a lot of references reading and investigation researches, the design of the process of venue booking functions are as follows:

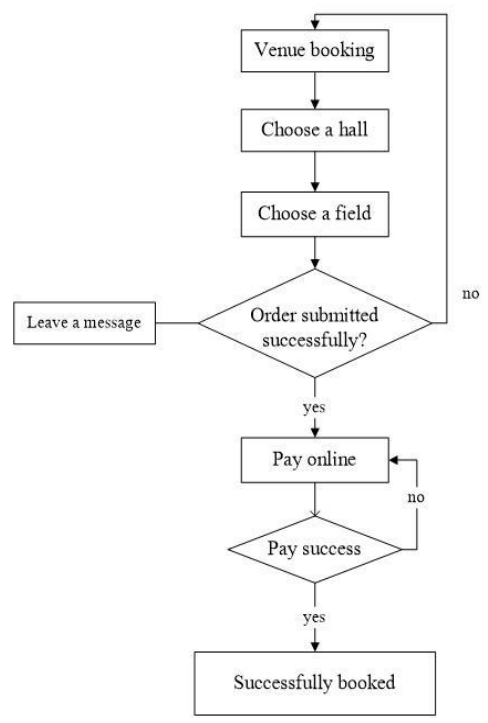

Fig. 5. Flow chart of venue booking.

Take table tennis hall as an example, the venues' distribution plan and its real-time information are shown to users in the form of a plane. The design is reasonable and seems to be clear at a glance. The user can booking venues within seven days from now on site, and the distribution of the time period is divided into 90 minutes a time period one day, They are 8:00-9:30,10:00-11:30 and other time periods in the afternoon. After a click, it can jump to the pay function page. If the payment is successful, the reservation can be achieved. 


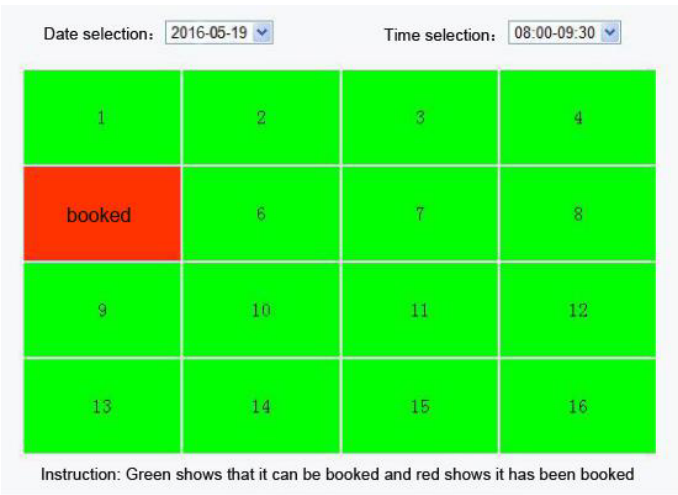

Fig. 6. Booking interface.

\subsection{Design and implementation of payment function}

The payment function is another difficulty of this study. To implement a online payment, the virtual account was introduced. Through deducting the user's account points at the time of booking site, the payment was achieved. When submitting an order, users will be asked if they do determine the venue booking. If they click yes, their accounts will be deducted, and then the venue booking is succeed. Users can view their orders or cancel them in a column called "my orders" in the personal center module.

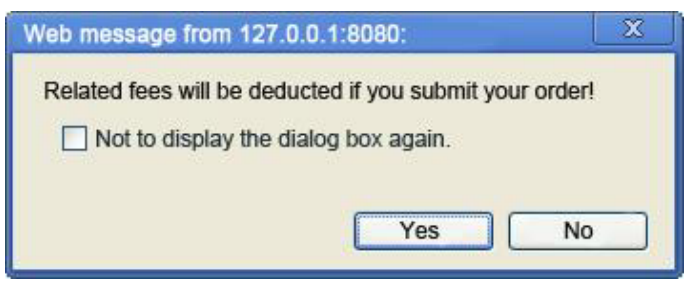

Fig. 7. Prompt of deduction.

\subsection{Design and implementation of message board function}

When submit orders, users can leave a message to the administrator if they have more demands, and then the administrator should meet users' demands as far as possible. Which can strengthen communication and exchange of information, making our services more thoughtful. Moreover, this is in accordance with the humanization design concept of the system.

Leave a message: \begin{tabular}{|l|} 
Wish a good service! \\
Submit order Cancel
\end{tabular}

Fig. 8. Message board.

After success of submit orders:

\begin{tabular}{cccc}
\hline User name & Note & Status & Operation \\
lican & Wish a good service! & Paid & Cancel the order
\end{tabular}

Fig. 9. Display of message.

\subsection{Design and implementation of personal center module}

(1) Personal center module for ordinary users

It is mainly divided into two parts, they can view or cancel their order here. The second is a personal information module, the user can not only change or improve their personal information, but also change password, etc. 


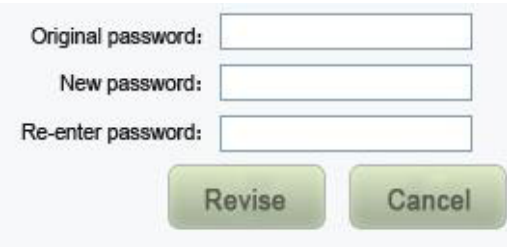

Fig. 10. Change password.

(2)Personal center module for administrator users

Besides with normal ordinary user' permissions, administrator uses have more functions which can mainly divided into the following three aspects: Member management, bulletin management and order management. Administrator users can implement a change of information of bulletin board, they can check all users' orders, change or delete ordinary users' information and recharge accounts for users, etc.

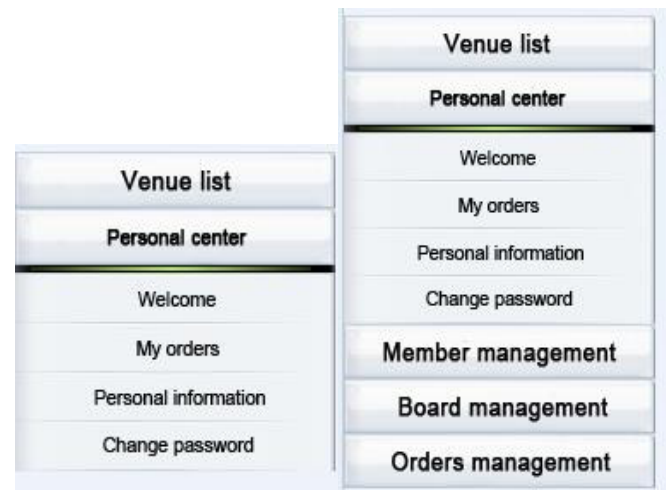

Fig. 11. Different permissions between ordinary users(left) and administrator users(right).

\subsection{Construction of the database}

The system mainly used a data access model named JDBC to access the data resources of database MySQL server. Several major tables are as follows:

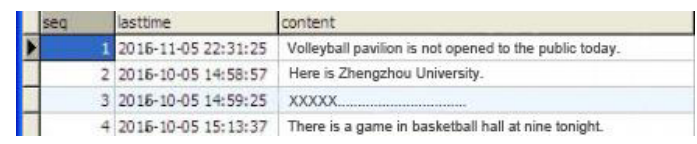

Fig. 12. Bulletin board information table.

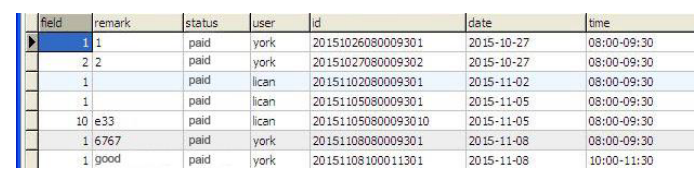

Fig. 13. Orders information table.

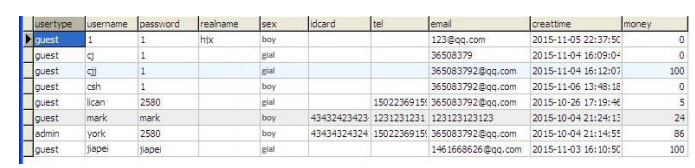

Fig. 14. Users information tabl.

\section{Test and improvement of the system}

\subsection{Test of browser's compatibility}


There are many different versions of browsers, so the browser's compatibility must be maintained to make sure the system are correctly displayed under different browsers. IE Tester can be used to test various versions of IE browsers' effects of the same system. Such browsers as IE6, IE7, IE8, firefox, Google and Opera are installed to test the system. Finally, the tests are all passed.

\subsection{Test of operation of the system}

Alpha and Beta are respectively used to make a internal and external tests. We find problems existed in the system and constantly improve it by summarzing test results.

\section{Conclusion and prospect}

The use of the system can made up for the current luck of sports venues management; It can solve the problems of the online booking function of university sports venues; It can improve the efficiency of the venues and meet the needs of efficient use of the venues. In this study, there are a lot of shortcomings. For instance, there is no limit to the number of reservation; It failed to set up a punishment and give a reasonable credentials into the venues. In the later study, the system needs completely improved. The order number, place number and the time may be feedbacked to users by moblephone messages in the future. The system has a great deal of portability.

The website can not only be bordered with web sites of school resources management, improving the management system, but also be extended to other sports venues of zhengzhou city or other colleges and universities, improving the use of stadiums resources.

\section{References}

1. W. Wang, W. Sun, http://news.xinhuanet.com/mrdx/2010-05/01/content_13451241.htm.

2. X. Dai, N. Zhang, Com. Mod. 11, 123-126 (2012).

3. X. Dai, N. Zhang, Int. Thin. Tech. 7, 69-71(2012).

4. H. Wei, Research and development of network service management platform of university sports facilities based on.NET Technology(Sichuan Normal University, 2011).

5. H. Zhang, The design and implementation of the management system of comprehensive sports facilities in Guizhou Normal University(Xiamen University, 2013).

6. C. Xia, J. Wuhan Eng Inst, 2, 48-50(2013).

7. R.Yuan, DIV + CSS Layout Essence of the Case( Tsinghua University Press, 2011).

8. H. Kong, X. Lu, Web Java design and practical tutorial - based on the application development of Eclipse environment(China Machine Press, 2012).

9. J. Zhao, Design and implementation of mobile business hall charging system based on MYECLIPSE development tools(University of Electronic Science and Technology of China, 2012).

10. C. Feng, Y. Chao, Tomcat and Java Web development Technology Description(Tsinghua University Press, 2008) 